\title{
METODOLOGÍAS DE ANÁLISIS AMBIENTAL Y VALORACIÓN DE LOS EFECTOS DE LA MINERÍA EN EL ENTORNO GLOBAL
}

\author{
Methodologies of environmental analysis and assessment \\ Of the effects of mining in the global environment
}

\section{EPISTEMUS}

ISSN: 2007-8196 (electrónico)

ISSN: 2007-4530 (impresa)

DR. Sergio A. Moreno Zazueta 1

MC. Juan M. Rodríguez Zavala ${ }^{2}$

Recibido: 10 de Abril de 2017,

Aceptado: 15 de Septiembre de 2017

Autor de Correspondencia:

DR. Sergio A. Moreno Zazueta

Correo:ocontreras@userena.cl

\section{Resumen}

Es innegable que la minería tiene un efecto significativo en el medio en el que se asienta. La minerígeneradora de desechos, en diversas etapas de sus procesos, emite e impacta al medio físico, causando efectos fácilmente identificables en los sitios en los que se asienta, sin embargo, los efectos a nivel global pueden analizarse desde una forma conceptual englobados en 7 grandes temas: Grandes volúmenes de desecho, Planificacción del cierre de minas, Legados de la minería, manejo ambiental. Uso de energía en el sector de los minerales, Manejo ambiental de los metales, Amenazas a la diversidad biológica.

Por ello se hace necesaria la utilización de las herramientas metodológicas de valoración ambiental aplicadas a la minería, debido a que este Sector industrial frecuentemente es tachado de "depredador del medio ambiente", la mayoría de las veces sin razón, o al menos sin un análisis serio de los impactos y efectos que está generando al medio físico. El uso de estas herramientas proporcionará una visión al empresario consciente, de las medidas aplicables al proceso para disminuir dichos efectos.

\section{Abstract}

It is undeniable that mining has a significant effect on the environment in which it is based. The waste minerígeneradora, in various stages of its processes, emits and impacts the physical environment, causing easily identifiable effects in the sites in which it is based, however, the global effects can be analyzed from a conceptual form encompassed in 7 large Topics: Large volumes of waste, Planning of mine closure, Legacies of mining, environmental management. Energy use in the minerals sector, Environmental management of metals, Threats to biological diversity.

Therefore it is necessary to use the methodological tools of environmental assessment applied to mining, because this industry is often labeled as "environmental predator", most of the time without reason, or at least without an analysis serious of the impacts and effects that it is generating to the physical environment. The use of these tools will provide a vision to the conscious entrepreneur, of the measures applicable to the process to reduce said effects. 


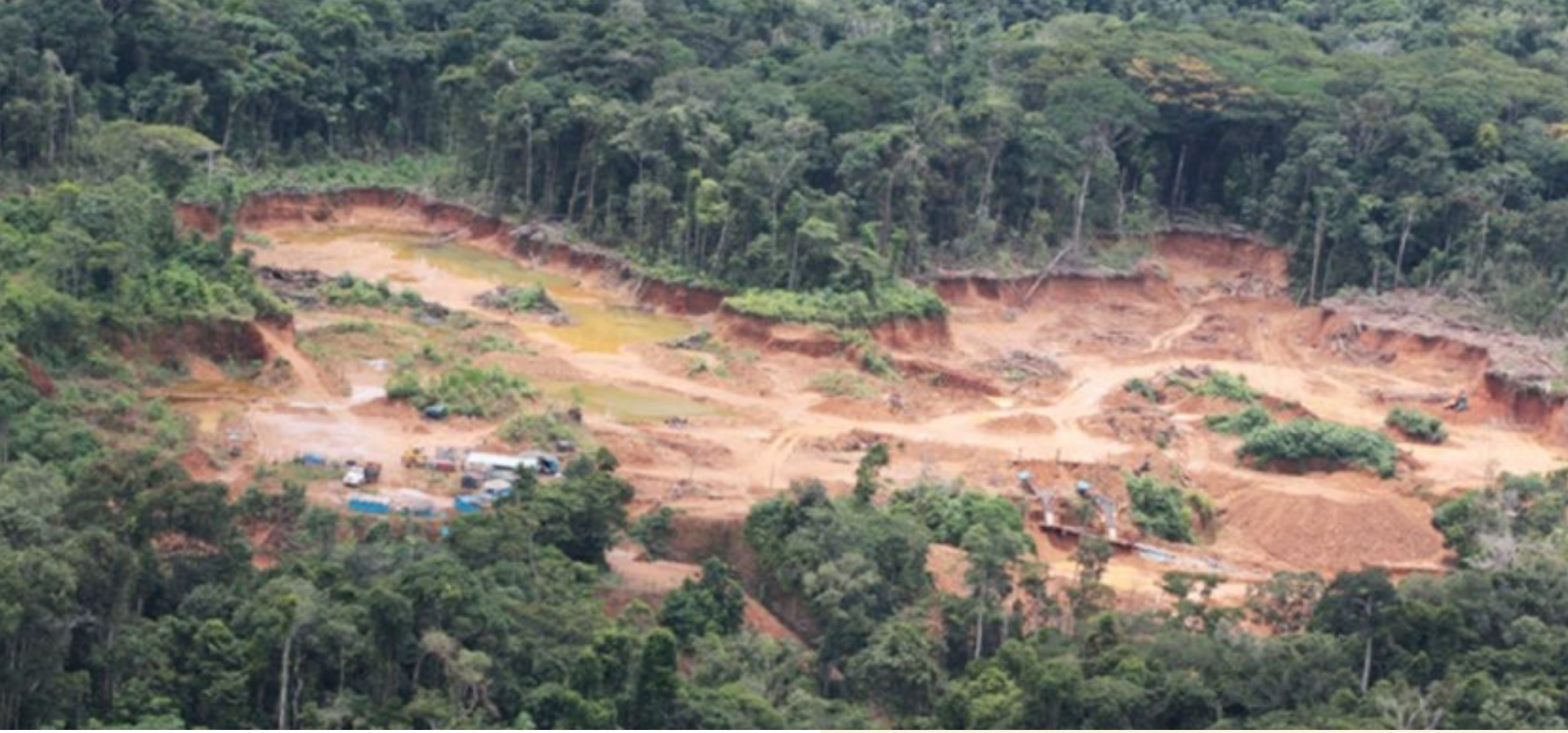

\section{HERRAMIENTAS CONCEPTUALES PARA LA GESTIÓN MEDIOAMBIENTAL}

Todos los sistemas de producción, procesos o servicios poseen un ciclo de vida que puede estructurarse de forma sistemática, con un principio y un final previamente establecido. En general, este ciclo de vida, que está compuesto por varios subsistemas conectados entre sí en forma de flujo progresivo, se inicia con la adquisición de las materias primas, pasando por otros subprocesos intermedios, hasta llegar al final de su vida útil cuando son llevados a vertedero.

La necesidad de estudiar, desde el punto de vista medioambiental, todas estas interrelaciones exigirán el empleo de métodos fiables que cuantifiquen o valoren todas estas acciones y sus efectos. Así, a la hora de tratar este tema es necesario proporcionar las respuestas adecuadas para atender a los objetivos esperados, entonces habrá que emplear herramientas que permitan medir los diversos tipos de parámetros, tanto aquellos clasificados de cuantificables como los de difícil cuantificación.

Entre los parámetros cuantificables están incluidos los relacionados con el consumo de materias primas, consumo de aguayenergía, emisiones de efluentes líquidos, emisiones de gases a la atmósfera, residuos sólidos, generación de co-productos, etc. Estos parámetros, pueden ser tratados a través de modelos, como por ejemplo, los de la base conceptual del análisis del ciclo de vida. Mientras los de difícil cuantificación, por ejemplo, los riesgos potenciales, cambios geográficos, impactos visuales del entorno o escasez de recursos son tratados con otras herramientas desarrolladas para tal fin. (2)

En las tablas 1 y 2 se muestran algunas de las principales herramientas hoy disponibles para la gestión medioambiental de sistemas de producción o productos y características básicas. (6)

Tabla 1. Herramientas conceptuales similares usadas en los sistemas de gestión ambiental ([SETAC, 1999).

\begin{tabular}{|l|l|}
\hline \multicolumn{1}{|c|}{ Herramienta } & \multicolumn{1}{c|}{ Traducción } \\
\hline RA - Risk Assessment & Análisis de riegos ambientales \\
\hline EIA - Environmental Impact Assessment & Estudio de impacto ambiental \\
\hline EAu - Environmental Auditing & Auditoría Ambiental \\
\hline EPE - Environmental Performance Evaluation & Evaluación del comportamiento ambiental \\
\hline SFA - Substance Flow Análisis & Análisis del flujo de sustancias \\
\hline EMA - Energy and Material Analysis & Análisis de material y energía \\
\hline ISCM - Integrated Substance Chain Management & Gestión integral de sustancias \\
\hline PLA - Product Line Analysis & Análisis de línea de producto \\
\hline LCA - Life Cycle Assessment & Análisis del ciclo de vida \\
\hline
\end{tabular}

Fuente: SETAC 
Tabla 2. Aspectos generales de las herramientas para la gestión ambiental. Fuente: SETAC

\begin{tabular}{|c|c|c|c|}
\hline $\begin{array}{l}\text { Análisis de riesgos } \\
\text { ambientales }\end{array}$ & $\begin{array}{l}\text { Valorar los efectos adversos } \\
\text { asociados a una situación específica } \\
\text { de riesgo y sus interrelaciones con la } \\
\text { salud humana y el medio ambiente. }\end{array}$ & $\begin{array}{l}\text {-Evalúa los efectos locales y } \\
\text { regionales bajo condiciones } \\
\text { específicas. }\end{array}$ & $\begin{array}{l}\text { - Es capaz de consumir mucho } \\
\text { tiempo y recursos. } \\
\text { - No es capaz de apuntar la ubicación } \\
\text { del riesgo a lo largo del ciclo de vida. }\end{array}$ \\
\hline $\begin{array}{l}\text { Estudio de impacto } \\
\text { ambiental }\end{array}$ & $\begin{array}{l}\text {-Evalúa los impactos positivos y } \\
\text { negativos sobre el medio ambiente } \\
\text { de un determinado proyecto } \\
\text { planteado. }\end{array}$ & $\begin{array}{l}\text {-Calcula tanto efectos positivos } \\
\text { como negativos. } \\
\text {-Considera los efectos locales } \\
\text { de un proyecto. }\end{array}$ & $\begin{array}{l}\text {-No es capaz de apuntar fácilmente } \\
\text { la ubicación de un efecto global/ } \\
\text { regional u otros efectos a lo largo del } \\
\text { ciclo de vida. }\end{array}$ \\
\hline Auditoría ambiental & $\begin{array}{l}\text { - Verifica la conformidad con } \\
\text { determinados requisitos de } \\
\text { normativos vigentes, por medio de } \\
\text { chequeo realizado por tercera parte. }\end{array}$ & $\begin{array}{l}\text { - Proporciona una manera } \\
\text { para que una tercera parte, } \\
\text { independiente, compruebe los } \\
\text { resultados. }\end{array}$ & $\begin{array}{l}\text { - Enfoca una conformidad y enfatiza } \\
\text { en término medio de más débil que } \\
\text { de mejoría. }\end{array}$ \\
\hline $\begin{array}{l}\text { Evaluación del } \\
\text { comportamiento } \\
\text { ambiental }\end{array}$ & $\begin{array}{l}\text { - Proporciona una información fiable, } \\
\text { objetiva y comprobable a cerca del } \\
\text { desempeño medioambiental de una } \\
\text { determinada organización. }\end{array}$ & $\begin{array}{l}\text { - Proporciona coeficientes } \\
\text { de desempeño } \\
\text { medioambiental asociándolos } \\
\text { a políticas objetivas y metas } \\
\text { preestablecidas. }\end{array}$ & $\begin{array}{l}\text {-Promociona coeficientes de } \\
\text { desempeños relativos y no absolutos. }\end{array}$ \\
\hline $\begin{array}{l}\text { Análisis del flujo de } \\
\text { sustancias }\end{array}$ & $\begin{array}{l}\text {-Contabilizar el suministro y } \\
\text { la demanda de una sustancia } \\
\text { específica que fluye a través del } \\
\text { proceso de producción }\end{array}$ & $\begin{array}{l}\text { - Toma en consideración un } \\
\text { impacto potencial determinado } \\
\text { a lo largo del ciclo de vida. }\end{array}$ & $\begin{array}{l}\text { - El enfoque sobre una única } \\
\text { sustancia puede apuntar falsos } \\
\text { resultados. }\end{array}$ \\
\hline $\begin{array}{l}\text { Análisis de material } \\
\text { y energía }\end{array}$ & $\begin{array}{l}\text { - Calcular el balance energético y } \\
\text { material asociado con una operación } \\
\text { específica. }\end{array}$ & $\begin{array}{l}\text { - Promociona una vía } \\
\text { estructurada de identificación } \\
\text { y valoración de un impacto } \\
\text { potencial de operaciones, etc. }\end{array}$ & $\begin{array}{l}\text { - Enfoca solamente una fases del } \\
\text { ciclo de vida. }\end{array}$ \\
\hline $\begin{array}{l}\text { Gestión integral de } \\
\text { sustancias }\end{array}$ & $\begin{array}{l}\text { - Calcular y reducir globalmente el } \\
\text { impacto medioambiental de una } \\
\text { determinada sustancia asociada. }\end{array}$ & $\begin{array}{l}\text { - Permite hacer consideraciones } \\
\text { entre económicas y } \\
\text { medioambientales en una } \\
\text { misma herramienta. }\end{array}$ & $\begin{array}{l}\text { - Emplea una valoración simplificada } \\
\text { que puede dar respuestas demasiado } \\
\text { simplificadas. }\end{array}$ \\
\hline
\end{tabular}

Fuente: SETAC

\section{EFECTOS DE LA MINERÍA EN EL ENTORNO GLOBAL}

Es innegable, como ya lo hemos mencionado, que la minería tiene un efecto significativo en el medio en el que se asienta. Es en el medio físico donde sus impactos son más notorios; sin embargo, vale la pena definir brevemente las condiciones y características propias de estas interacciones con dicho entorno.

\section{La minería como generadora de desechos}

Los productos minerales y otros recursos minerales rara vez son encontrados en un estado lo suficientemente puro como para ser comercializados tal como se minan. En el caso de los metales, frecuentemente son encontrados en combinaciones químicas con el oxigeno (óxidos), con el azufre (sulfuros) o con otros elementos (arseniatos, cloruros, carbonatos, fosfatos etc.). Los no-metálicos contienen impurezas - algunas veces ligadas físicamente y otras químicamente- que también obligan a su "purificación".

La mayor fuente de residuos sólidos generados por la minería y los subsecuentes procesos es la ganga (minerales sin valor o valor no económico asociado con el mineral de interés). Dependiendo de la etapa del proceso en que esta "ganga" es desechada, recibe diferentes denominaciones: roca estéril cuando es separada en el proceso de minado, jales o lodos de molienda cuando lo es durante la etapa de procesamiento del mineral, escoria cuando es separada durante los procesos pirometalúrgicos y muchos otros nombres (polvos, slurries, pilas de mineral lixiviado, etc.) 
En las tablas 3 y 4 se resume los principales impactos de las actividades minero-metalúrgicas.

Tabla 3. Procesos específicos por sector de la minería metálica y residuos generados

\begin{tabular}{|c|c|c|c|}
\hline Sector & Tipo de Minado & $\begin{array}{l}\text { Proceso de Beneficio/ } \\
\text { Concentración }\end{array}$ & $\begin{array}{c}\text { Residuos y/o emisiones } \\
\text { principales }\end{array}$ \\
\hline Oro-Plata-PGM & $\begin{array}{l}\text { Superficial } \\
\text { Subterráneo } \\
\text { In Situ (experimental) }\end{array}$ & $\begin{array}{l}\text { Cianuración } \\
\text { Elusión } \\
\text { Electrólisis } \\
\text { Molienda } \\
\text { Flotación } \\
\text { Fundición } \\
\text { Amalgamación (histórica) }\end{array}$ & $\begin{array}{l}\text { Agua de Mina } \\
\text { Roca estéril } \\
\text { Solución estériles de } \\
\text { proceso } \\
\text { Jales } \\
\text { Mineral Tratado }\end{array}$ \\
\hline Plomo- Zinc - Níquel & Subterráneo & $\begin{array}{l}\text { Molienda } \\
\text { Flotación } \\
\text { Fundición } \\
\text { Sinterización }\end{array}$ & $\begin{array}{l}\text { Agua de Mina } \\
\text { Roca estéril } \\
\text { Jales } \\
\text { Escoria de fundición }\end{array}$ \\
\hline $\begin{array}{l}\text { Hierro - Titanio- Cromo } \\
\text { - Manganeso }\end{array}$ & $\begin{array}{l}\text { Superficial } \\
\text { Subterráneo }\end{array}$ & $\begin{array}{l}\text { Molienda } \\
\text { Separación Magnética } \\
\text { Gravimetría } \\
\text { Flotación } \\
\text { Aglomeración } \\
\text { Medios densos } \\
\end{array}$ & $\begin{array}{l}\text { Agua de Mina } \\
\text { Roca estéril } \\
\text { Jales } \\
\text { Escoria }\end{array}$ \\
\hline Aluminio & $\begin{array}{l}\text { Superficial } \\
\text { Subterráneo }\end{array}$ & $\begin{array}{l}\text { Molienda } \\
\text { Medios densos }\end{array}$ & $\begin{array}{l}\text { Agua de Mina } \\
\text { Roca estéril }\end{array}$ \\
\hline
\end{tabular}

Fuente: modificada U S. E. P. A.

Tabla 4. Operaciones unitarias de la minería e impactos principales

\begin{tabular}{|c|c|c|c|c|}
\hline Proceso & Residuos del Proceso & Emisiones al Aire & Otros residuos & Suelo, Ecosistema \\
\hline $\begin{array}{l}\text { Preparación del } \\
\text { Sitio }\end{array}$ & $\begin{array}{l}\text { Erosión debida a } \\
\text { la remoción de la } \\
\text { vegetación }\end{array}$ & $\begin{array}{l}\text { Gases de los } \\
\text { vehículos de } \\
\text { construcción, polvo }\end{array}$ & $\begin{array}{l}\text { Sedimentos a } \\
\text { vías de agua }\end{array}$ & Deforestación \\
\hline $\begin{array}{l}\text { Barrenación y } \\
\text { voladura }\end{array}$ & $\begin{array}{l}\text { Drenaje Acido de } \\
\text { Mina (DAM); erosión } \\
\text { de sedimentos, } \\
\text { residuos de aceites de } \\
\text { vehículos }\end{array}$ & $\begin{array}{l}\text { Polvo, gases de } \\
\text { maquinaria pesada }\end{array}$ & Roca estéril & $\begin{array}{l}\text { Incremento de erosión, pérdida de } \\
\text { flora y fauna, reducción de captación } \\
\text { de acuíferos, daños estructurales por } \\
\text { vibración, competencia de uso de } \\
\text { suelo. }\end{array}$ \\
\hline $\begin{array}{l}\text { Procesos de } \\
\text { reducción de } \\
\text { Tamaño }\end{array}$ & & Polvo & $\begin{array}{l}\text { Roca estéril } \\
\text { adicional }\end{array}$ & \\
\hline $\begin{array}{l}\text { Procesos de } \\
\text { Separación de } \\
\text { Minerales }\end{array}$ & DAM & Polvos & Jales & $\begin{array}{l}\text { Impacto al paisaje, contaminación de } \\
\text { acuíferos }\end{array}$ \\
\hline Lixiviación & $\begin{array}{l}\text { DAM, pilas de roca } \\
\text { estéril }\end{array}$ & & $\begin{array}{l}\text { Lodos de } \\
\text { purgado }\end{array}$ & $\begin{array}{l}\text { Afecciones a la fauna por } \\
\text { contaminación de aguas }\end{array}$ \\
\hline
\end{tabular}

Fuente: modificada U S. E. P. A. 

física como estabilidad socioeconómica debería ser parte fundamental del ciclo de vida del proyecto y debería ser diseñado para asegurar que:

- No se comprometa la salud ni la seguridad pública a futuro

- Los recursos ambientales no estén expuestos a deterioro físico ni químico

- El uso posterior del recinto sea beneficioso y sostenible en el largo plazo

- Cualquier impacto socioeconómico sea reducido al mínimo

- Todos los beneficios socioeconómicos sean maximizados

\section{c) Legados de la minería}

Los problemas ambientales de las actuales y futuras operaciones mineras son lo suficientemente desalentadores. Pero en muchas maneras mucho más problemáticos son algunos de los continuos efectos de la extracción y fundición que han tenido lugar a través de las últimas décadas, siglos e incluso milenios. Estas zonas mineras han comprobado que algunos impactos pueden ser de largo plazo y que la sociedad aún está pagando el precio de los recursos del capital natural que han sido agotados por las generaciones pasadas.

En la mayoría de los países con larga historia minera, existe relativamente poca información sobre minas antiguas o su legado ambiental, aunque la información es suficiente para saber que los problemas son generalizados. (1).

La primera prioridad global para las autoridades públicas debe ser identificar y registrar las minas abandonadas y evaluar el riesgo que éstas constituyen. Dada la magnitud del problema y la capacidad limitada de las oficinas públicas, se deberá establecer prioridades -el proceso de registro, por ejemplo, debería ser fijado más allá de algún acuerdo con respecto al tamaño de la mina.

La segunda prioridad en el ámbito nacional e internacional, debería ser desarrollar nuevos mecanismos de financiamiento que fueran los suficientemente sólidos y sostenibles para hacer frente a los problemas que serán una carga para las futuras generaciones.

\section{d) Manejo ambiental}

Para obtener todos los beneficios que presenta una Evaluación de Impacto Ambiental, ésta debería formar parte de un Sistema de Manejo Ambiental (SMA) que busque integrar responsabilidades ambientales en las prácticas de manejo diarias a través de cambios de la estructura, responsabilidades, procedimientos, procesos y recursos de la organización.

Un SMA proporciona a la empresa un método estructurado de manejo y entrega a la autoridad reguladora la conciencia y el control con respecto al desempeño de un proyecto que puede ser aplicado en todas las etapas del ciclo de vida - desde la identificación de un yacimiento hasta el cierre de la mina. Las etapas de un sistema de manejo ambiental son las siguientes:

- Compromiso organizativo

- Política ambiental

- Evaluación de impacto socioeconómico

- Evaluación de impacto ambiental

- Consulta a la comunidad

- Objetivos y metas

- Plan de manejo ambiental

- Manual ambiental y de documentación,

- Procedimientos de emergencia y control operativo

- Capacitación

- Seguimiento de emisiones y desempeño

- Auditorías ambientales y de cumplimiento

- Revisiones

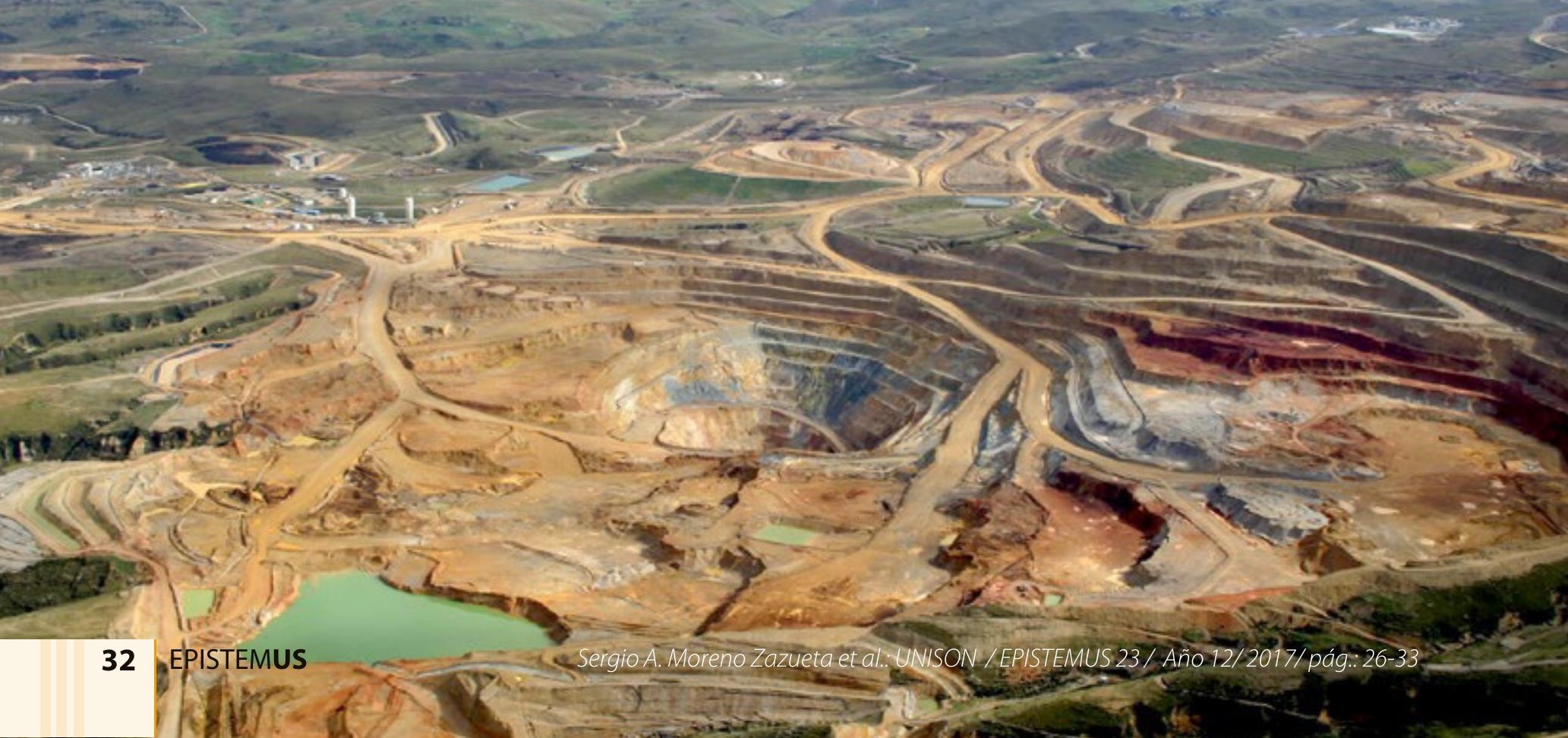




\section{e) Uso de energía en el sector de los minerales}

El actual nivel y modelo de uso de energía es un factor fundamental que afecta las condiciones ambientales del mundo; el cambio climático es una preocupación crucial para el desarrollo sostenible. Este cambio climático tiene la posibilidad de provocar impactos graves a la reproducción de los ecosistemas y una vez que se produce es difícil revertir.

Existen varias razones por las cuales el sector de los minerales se encuentra especialmente involucrado en los aspectos de un potencial cambio ambiental global que se relacionan con el uso de energía:

- La elaboración de productos minerales básicos a partir de fuentes primarias implica el traslado y el procesamiento de grandes cantidades de material, lo que requiere una fuente de energía.

- Muchos productos elaborados que dependen de los insumos minerales para su funcionamiento consumen cantidades considerables de energía, como por ejemplo vehículos motorizados y artículos eléctricos.

- Debido a los requisitos de energía, la minería y la industria de los minerales pueden influir las decisiones con respecto a invertir en fuentes de energía.

- Varios productos minerales básicos, cabe destacar el caso del carbón, son utilizados como combustibles.

Aunque a veces se dijo que entre el 4 y el $7 \%$ de la demanda de energía del mundo es consumida por la minería, los límites no están lo suficientemente definidos para determinar una cifra mundial exacta. (3).

\section{f) Manejo ambiental de los metales}

Varios metales generan gran preocupación ambiental debido a su potencial toxicidad química. Estos temores se extienden a los metaloides -elementos no metálicos, como por ejemplo el arsénico, y que en algunos aspectos se comportan como metales. De hecho, las propiedades tóxicas de muchos metales y metaloides han sido explotadas para diseñar pesticidas y antisépticos.

Para muchas personas, el miedo a su toxicidad es tan importante como el daño que se sabe, han causado estos elementos. Este es un problema importante con respecto a la comunicación de riesgos y puede tener consecuencias sociales y económicas.

\section{g) Amenazas a la diversidad biológica}

El sector de los minerales tiene un papel fundamental en el mantenimiento de la biodiversidad, ya que algunas operaciones mineras pueden eliminar ecosistemas completos, todas sus especies endémicas y hacer que sus actividades sean cada vez más prolíficas en áreas relativamente inalteradas y de alto valor de biodiversidad. Sin embargo, el éxito duradero dependerá de acciones de rehabilitación por parte de todos los sectores, incluyendo planificación económica, agricultura, pesca, energía, infraestructura y turismo. También dependerá de la comprensión que tengan los consumidores más ricos sobre el impacto social y ecológico que generan sus modelos de consumo.

\section{CONCLUSIONES}

Cada vez es más necesaria la utilización de las herramientas metodológicas de Valoración ambiental aplicadas a la minería, debido a que este Sector industrial frecuentemente es tachado de "depredador del medio ambiente", la mayoría de las veces sin razón, o al menos sin un análisis serio de los impactos y efectos que está generando al medio físico.

El uso de estas herramientas proporcionará una visión al empresario consciente, de las medidas aplicables al proceso para disminuir dichos efectos.

\section{BIBLIOGRAFÍA}

1. Ashton, P J, Love, D,Mahachi, H, y Dirks, P (2001) "An Overview of the Impact of Mining and Mineral Processing Operations on Water Resources and Water Quality in the Zambezi, Limpopo and Olifants Catchments in Southern Africa". CSIR-Environmentek, Pretoria, Sudáfrica, y Departamento de Geología, Universidad de Zimbabwe, Harare, Zimbabwe. Informe preparado para MMSD Sur de África.

2. Johansson, J. (1999). "A monetary Valuation Weighting Method for Life Cycle Assessment Based on Environmental Taxes and Fees" Master Thesis in Ntural Resource Management. Stockholm University, Sweden.

3. Lovins,A B, Feiler,T E, y Rábago, K R (2002) “Energy and Sustainable Development in the Mining and Minerals Industries". Informe preparado para MMSD.

4. Moreno, S. (2007) "Valoración de las Cadenas Productivas de la Minería Metálica Global, usando Herramientas innovadoras de Gestión Ambiental" Tesis Doctoral UPM. España.

5. Phelps, R W (2000) “Moving a Mountain a Day - Grasberg Grows Six-Fold". Engineering \& Mining Journal, $1^{\circ}$ de junio, 2000. págs. 22-28.

6. SETAC (1999) "Life cycle assessment and conceptually Related Programmes". Europe Working Group, Brussels Belgium

7. World Resources Institute (2000): "Recursos Mundiales 2000. Guía Global de Medio Ambiente", Editorial Ecoespaña, Madrid. 